\title{
Swift Communications and Public Outreach
}

\author{
L.Cominsky* \\ Sonoma State University \\ E-mail: Iynnceuniverse.sonoma.edu
}

\section{A. Simonnet}

Sonoma State University

E-mail: auroreduniverse.sonoma.edu

and the Swift E/PO team

The Sonoma State University (SSU) Education and Public Outreach (E/PO) group participates in the planning and execution of press conferences that feature noteworthy Swift discoveries, as well as supporting social media and outreach websites. We have also created many scientific illustrations for the media, tools for amateur astronomers for use at star parties, and have given numerous public talks about Swift discoveries.

Swift: 10 Years of Discovery,

2-5 December 2014

La Sapienza University, Rome, Italy

\footnotetext{
* Speaker.
} 
Table 1: Media Releases and Briefings

\begin{tabular}{|c|c|c|}
\hline Year & Releases & Media Events \\
\hline 2004 & 2 & Launch Coverage (NASA) \\
\hline 2005 & 13 & $\begin{array}{l}\text { First Light, First afterglows, Superflare from SGR 1806-20 (NASA), } \\
\text { First redshifts, Most Distant GRB050904 (NASA), Short GRBs (AAS), } \\
\text { First short burst afterglow from GRB050509b (NASA) }\end{array}$ \\
\hline 2006 & 11 & $\begin{array}{l}\text { Unusual Type 1c GRB 060218, Supernova properties (HEAD), } \\
\text { Black Hole Census (HEAD), Hybrid GRB } 060614\end{array}$ \\
\hline 2007 & 8 & Flares after GRBs, Shot in the Dark GRB 070125 \\
\hline 2008 & 8 & $\begin{array}{l}\text { Distant Short GRB 070714B (AAS), Naked Eye GRB 080319B (NASA), } \\
\text { Supernova Breakout (Princeton), Most Distant GRB 080913, } \\
\text { Smallest Black Hole (HEAD) }\end{array}$ \\
\hline 2009 & 8 & $\begin{array}{l}\text { Bright GRB } 080607 \text { (AAS), Comet Observations (IYA), } \\
\text { Andromeda UVOT image (Blueshift), GRB } 080607 \text { in Star Factory (AAS), } \\
\text { Active Galaxies in Merging Systems (NASA), Dark GRBs (AAS) }\end{array}$ \\
\hline 2010 & 6 & $\begin{array}{l}\text { Swift's 500th Burst, Record-breaking Burst GRB 100621A, } \\
\text { Black Hole Survey (NASA) }\end{array}$ \\
\hline 2011 & 8 & $\begin{array}{l}\text { Tidal Disruption in Christmas Burst GRB 101225A, } \\
\text { iPhone App, Distant GRB 090429B (AAS) }\end{array}$ \\
\hline 2012 & 10 & UVOT Image Gallery, Changes in Exoplanet Atmosphere \\
\hline 2013 & 11 & $\begin{array}{l}\text { Ultra-long Bursts (GRB), Nearest luminous GRB 130427A, } \\
\text { LMC/SMC Mosaics (AAS), Anti-glitch in Magnetar 1E 2259+586 }\end{array}$ \\
\hline 2014 & 10 & $\begin{array}{l}\text { Center of Milky Way (AAS), Swift at } 10 \text { Years, } \\
\text { Kilonova Explosion Produces Gold in GRB 130603B }\end{array}$ \\
\hline
\end{tabular}

\section{Press Conferences and Press Releases}

Beginning with the activities leading up to the launch of Swift on November 20, 2004, there have been many press conferences, media telecons, press releases and news features that showcase the discoveries and news about Swift. Table $\square$ summarizes the number of news releases and features issued each year since launch, as well as each year's top stories. Many of these were showcased in press briefings or media telecons occurring at scientific conferences including: the American Astronomical Society (AAS) winter and summer meetings, the annual GRB Symposia (GRB), and the AAS High Energy Astrophysics Division (HEAD) meetings. Other media events occurred during the International Year of Astronomy (IYA), at Princeton University and through the NASA Blueshift podcast channel. For complete links see http://swift.gsfc.nasa.gov/news/.

\subsection{Science Magazine Covers}

A Swift discovery in 2013 was the subject of the 3 January 2014 cover story in Science magazine. An illustration by Aurore Simonnet depicted observations of GRB 130427A, one of the brightest gamma-ray bursts ever seen. 
Table 2: Swift SVS Videos with more than 75,000 views to date

\begin{tabular}{|l|r|r|c|}
\hline Video & Date & Views & SVS \# \\
\hline $\begin{array}{l}\text { NASA's Swift Provides the Best-Ever UV View } \\
\text { of the Nearest Galaxies }\end{array}$ & 3 Jun 2013 & $1,896,437$ & 11293 \\
\hline NASA's Swift Spots Black Hole Devouring A Star & 24 Aug 2011 & 335,238 & 10807 \\
\hline X-Ray Nova Reveals a New Black Hole in Our Galaxy & 5 Oct 2012 & 114,901 & 11108 \\
\hline Swift's UV portrait of the Andromeda Galaxy & 16 Sep 2009 & 107,727 & 10485 \\
\hline The Dual Personality of the 'Christmas Burst' & 30 Nov 2011 & 95,747 & 10808 \\
\hline A Trio of Swift Bursts Form A New Class of GRBs & 16 Apr 2013 & 84,729 & 11250 \\
\hline Swift: A Decade of Game-Changing Astrophysics & 20 Nov 2014 & 78,419 & 10171 \\
\hline
\end{tabular}

Table 3: Swift YouTube Videos with more than 50,000 views since 1 Jan 2009

\begin{tabular}{|l|r|r|r|}
\hline Video & Date & Views & YouTube \\
\hline $\begin{array}{l}\text { NASA's Swift Satellite Spots Black Hole } \\
\text { Devouring A Star }\end{array}$ & 24 Aug 2011 & $1,359,980$ & azLDH9ZPbVs \\
\hline Take a "Swift" Tour of the Andromeda Galaxy & 16 Sep 2009 & 456,379 & HWxBTHVhc3I \\
\hline A Swift Tour of the Nearest Galaxies in UV Light & 3 June 2013 & 180,787 & 53yokIKAnDs \\
\hline Swift Catches Mega Flares from a Mini Star & 30 Sep 2014 & 175,419 & hL9OHXw_-A8 \\
\hline Swift Captures Flyby of Asteroid 2005 YU55 & 10 Nov 2011 & 102,635 & vtEvuz_oQ5o \\
\hline $\begin{array}{l}\text { Swift's Christmas Burst from Blue } \\
\text { Supergiant Star Explosion }\end{array}$ & 16 Apr 2013 & 67,213 & MVQI8RC67Yw \\
\hline
\end{tabular}

\subsection{YouTube and SVS Videos}

NASA Goddard's Scientific Visualization Studio (SVS) employs many talented animators and illustrators that help explain Swift's scientific discoveries to the public. Many press briefings, media telecons, press releases and news features include illustrations and video products that help to explain Swift's visions of the Universe. Since launch, there have been about 4 million views of Swift videos on the SVS website (http://svs.gsfc.nasa.gov). Table $\square$ lists Swift SVS videos with more than 75,000 views, along with viewing statistics and the SVS reference number.

Beginning in 2009, animated media products that were created to illustrate press releases and briefings have been uploaded to NASA's YouTube channel. Table B lists Swift YouTube videos with more than 50,000 views, along with viewing statistics and the YouTube reference code.

\section{Public Outreach}

From the top of Mt. Tamalpais to seniors in the Osher Life Long Learning Institute to amateur astronomers across the USA, Swift E/PO lead Lynn Cominsky has given dozens of public lectures about Swift's View of the Universe. Others who have given many public talks include Swift Principal Investigator Neil Gehrels; team members John Nousek, Dirk Grupe, Brad Cenko, Jo-Anne 

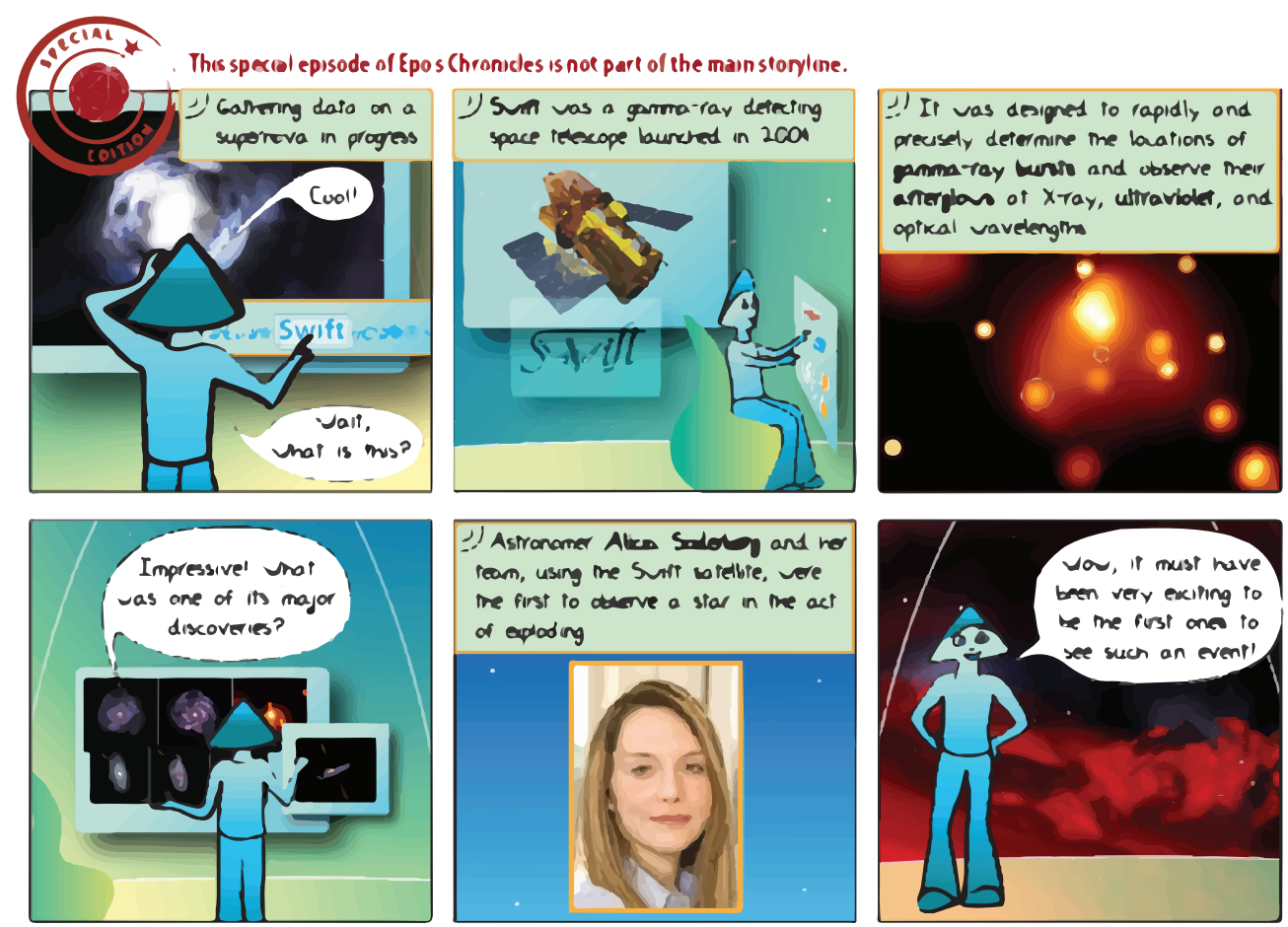

Figure 1: Epo's Chronicles special "eposode" about Swift's discovery of a supernova shock breaking out

Hill, and Padi Boyd; and observers Edo Berger, Alicia Soderberg, Andy Fruchter, Alex Filippenko, Derek Fox, and Suvi Gezari.

\subsection{Epo's Chronicles}

From 2008 - 2013, the SSU E/PO team produced over 200 weekly “eposodes” of Epo's Chronicles, a web comic that illustrated the adventures of Alkina and her sentient spaceship Epo. Alkina and Epo traveled through the galaxy, learning about space science and searching for their origins. Translated from English into French, Italian and Spanish, these popular comic strips were viewed by thousands each month. During 2012 (the last complete year of the webcomic), over 80,000

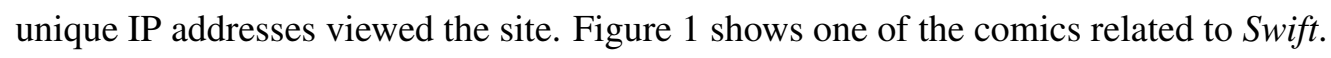

\subsection{Amateur Astronomers}

Public outreach is often conducted by amateur astronomers through star parties held nationwide. The Swift E/PO team co-sponsored the SUPERNOVA! toolkit for use by these passionate advocates for astronomy. Since 2008, when the toolkit went into national distribution, it has reached over 138,000 attendees through more than 1300 events. Of these events, 680 events reported including almost 25,000 minorities and over 39,000 women/girls.

\subsection{GRB Skymap Website}

The Gamma-ray Burst Skymap website http://grb. sonoma. edu was actively maintained during 2004 - 2013. The site automatically updates for each gamma-ray burst as it occurs, 
whether detected by Swift or other orbiting satellites. For each burst, the location on the sky, starmap, constellation and detecting mission are generated automatically. It was then quickly updated by hand to include a written description of the burst properties and scientific significance, as observations continue. As of 2014, burst descriptions are no longer being written, but some GCN burst locations update automatically. During 2012, a total of 34,428 unique IP addresses accessed the site. An evaluation of the GRB Skymap website was conducted by external evaluators at WestEd in 2010. The study found that "The overall reaction to the GRB site from study participants was very positive. Each of the four participants had unique feedback, providing information about how different types of users view the site. The Gamma-ray Burst Real-time Sky Map is a powerful and informative site."

\subsection{Social Media}

Swift's presence in the world of social media includes a Facebook page and the Twitter feed @NASASwift. Since the Twitter feed was started in December, 2011, there have been 87 tweets from @NASASwift, and the feed has over 4,300 followers. The Swift Facebook page has over 9,600 likes and can be found at https://www. facebook. com/nasaswift.

\subsection{Swift GRB Lottery Game}

The Swift E/PO website includes a link to the GRB Lottery Game, in which members of the public try to guess the position of the next GRB to be detected by Swift. Winners of the Lottery Game are sent a "prize" of an outreach product that describes Swift. Since its debut in 2004, there have been about 400 winners. Figure $\square$ shows a screenshot of the Lottery game webpage at at http://swift.sonoma.edu/grb_lotto/index.php.

\subsection{International Year of Astronomy}

The year 2009 was the International Year of Astronomy (IYA). Public outreach events occurred throughout the world, and the Swift E/PO team participated in many of them, including the creation of special illustrated lithographs featuring the objects of the month as explained by Alkina from the Epo's Chronicles webcomic. Over 18,000 of these lithographs were distributed nationwide through amateur astronomy clubs through NASA's Night Sky Network. Another special creation was an Epo's Chronicles podcast distributed through the 365 Days of Astronomy website. This podcast was downloaded over 6000 times following its initial release on 16 September 2009. SSU E/PO created a traveling exhibit of IYA images that circulated around the San Francisco Bay Area during 2009. This small exhibit was featured at 20 venues, with an estimated viewing by over 100,000 participants. A larger IYA exhibit appeared at the California Academy of Sciences and San Jose Tech Museum, with estimated viewing of 50,000 at each location.

\subsection{Black Hole Planetarium Show}

The full-format digital dome planetarium show "Black Holes: The Other Side of Infinity" was produced in partnership with Fermi Gamma-ray Space Telescope, the National Science Foundation, the Denver Museum of Nature \& Science, and Tom Lucas Productions. Premiering in 2006, the show has been featured in over 30 venues and has reached millions of people world-wide. Narrated 


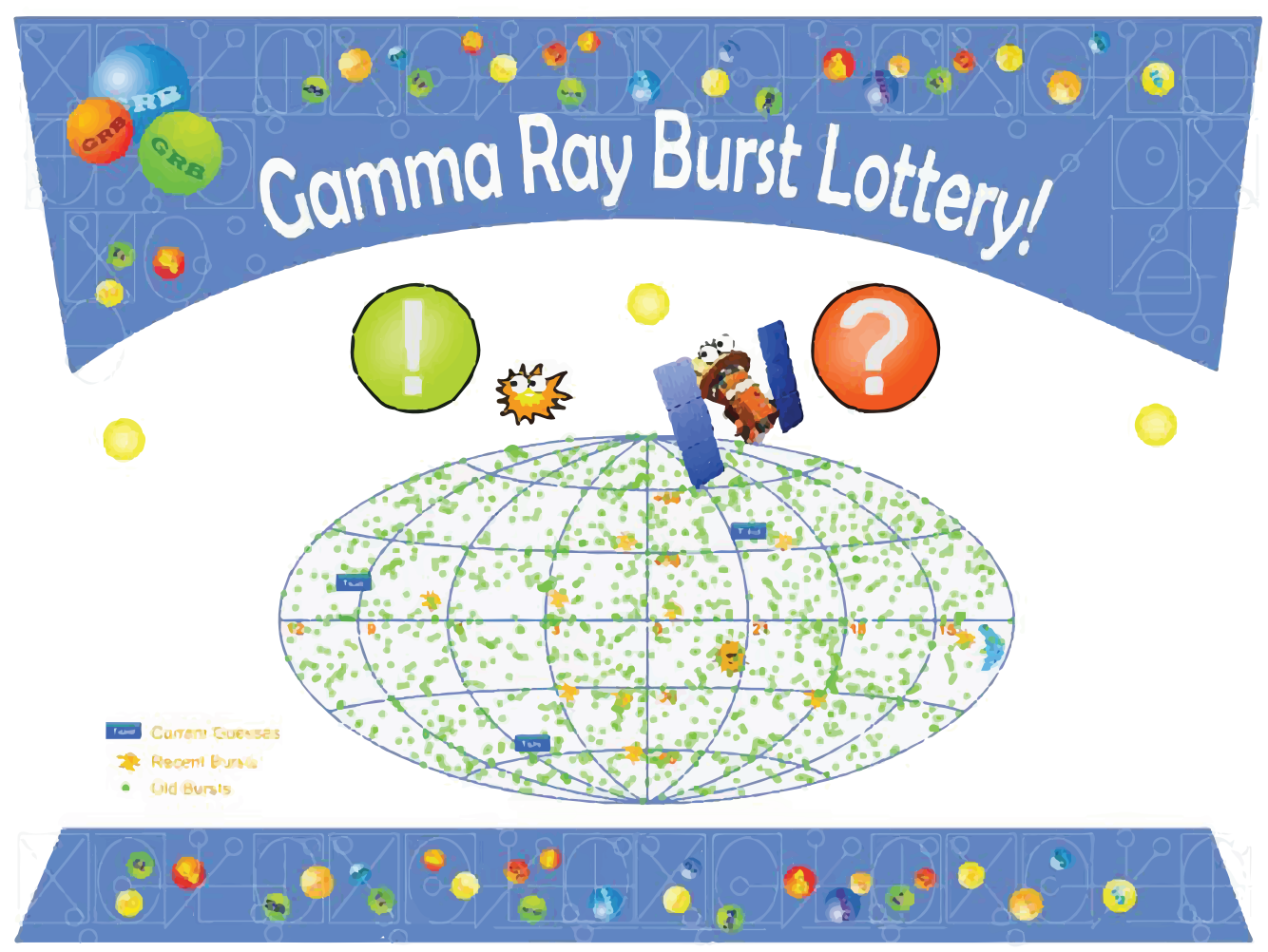

Figure 2: Screenshot of the Swift Lottery Game

by Liam Neeson, the opening scenes show the launch of Swift in November, 2004. The black hole show was initially seed-funded by Fermi E/PO and Lynn Cominsky served as a scientific director.

\subsection{Swift Newsletters}

During 2005 - 2009, Swift E/PO edited and published 11 newsletters for the public, featuring mission science discoveries. All newsletters appear online, but occasionally hard copies were also printed for distribution AAS meetings and other scientific conferences. Newsletters are archived at: http://swift. sonoma.edu/resources/multimedia/newsletter/index.html.

\subsection{Public Symposium at AAAS meeting}

Lynn Cominsky organized and moderated a scientific symposium at the AAAS meeting in February 2008 in Boston, Massachusetts, that showcased Swift science. Geared for (non-astronomer) scientific professionals, and other interested members of the public, this 90-minute symposium was entitled "The World-Wide Hunt To Solve The Mystery Of Gamma-Ray Bursts" and featured talks by Swift PI Neil Gehrels, UK Lead Alan Wells, Italian Lead Guido Chincarini and independent commentator Tsvi Piran from Hebrew University.

\subsection{Printed Materials}

Many printed materials were developed by the Swift E/PO team for public distribution. 
The Swift sticker features a colorful image of the satellite on the front along with text describing the mission on the back. Over 8000 of these were distributed.

The Swift lithograph features an image of the satellite on the front and on the back, describes the mission's scientific objectives, as well as providing an activity for students to do on the back.

The Swift fact sheet is a two-page color handout that briefly describes the Swift mission, its instruments, and ground system. Also included are tables listing the instrument parameters and a listing of the major institutions involved.

The Swift Glider is a paper model airplane designed to resemble the Swift bird, which also conveys information about the mission. Over 6500 gliders were distributed.

The Swift brochure "Catching Gamma-ray Bursts on the Fly" describes in detail the science that Swift does and explains how it does it. The description includes the three on-board instruments and their parameters, background information on gamma ray bursts and detection methods. It also describes pre-launch thinking about the origin of gamma-ray bursts.

The Swift paper model was based on the original version which was printed in Italian. It provides a short description of the scientific instruments on board the satellite, as well as links to other resources about its scientific objectives. It includes pages of printed parts that can be assembled into a paper model. About 2500 of these paper model booklets were distributed in English.

\subsection{Encyclopedia Articles}

Lynn Cominsky authored an article about Swift and gamma-ray bursts for the McGraw- Hill Yearbook of Science and Technology in July 2007. This article was updated in 2011 for the 2012 Yearbook. She also authored an encyclopedia article about Swift which appeared in Space Exploration and Humanity: A Historical Encyclopedia, published by the History Committee of the American Astronautical Society on August 23, 2010 with ISBN-10: 1851095144. In 2012, she also wrote an encyclopedia article about Gamma-ray bursts which includes Swift and which appears in the McGraw Hill Yearbook of Science and Technology, 2013 ISBN 978-0-07-180140-9.

\section{Acknowledgments}

The SSU Education and Public Outreach group would like to acknowledge support from NASA NNX07AF53G, NNX12AE34G, NNX12AK47G and NNX07AK19A. We also would like to thank the many talented science writers and animators at NASA Goddard Space Flight Center with whom we have worked during the years including Robert Gutro, Christopher Wanjek, Robert Naeye, Francis Reddy, Scott Wiessinger, and the staff at Goddard's Scientific Visualization Studio. 